\title{
An Overview of Massive MIMO Technology Components in METIS
}

\author{
Gábor Fodor $^{t t t}$, Nandana Rajatheva ${ }^{D}$, Wolfgang Zirwas ${ }^{\ddagger}$, Lars \\ Thiele $^{H}$, Martin Kurras ${ }^{H}$, Kaifeng Guo ${ }^{\times}$, Antti Töllii ${ }^{D}$, Jesper H. \\ Sorensen $^{q}$, Elisabeth de Carvalho ${ }^{q}$ \\ ${ }^{D}$ University of Oulu, Finland, E-mail: antti.tolli| rrajathe@ee.oulu.fi \\ ${ }^{7}$ Ericsson Research, Stockholm, Sweden. E-mail: gabor.fodor@ericsson.com \\ ${ }^{t t}$ Royal Institute of Technology, Stockholm, Sweden. E-mail: gaborf@kth.se \\ ${ }^{¥}$ Nokia Siemens Networks, Munich, Germany, E-mail: Wolfgand.Zirwas@nsn.com \\ ${ }^{H}$ Fraunhofer Heinrich Hertz Institute, Germany. E-mail: lars.thiele| martin.kurras@hhi.fraunhofer.de \\ ${ }^{\times}$RWTH Aachen University, Aachen, Germany, E-mail: Kaifeng.Guo@ice.rwth-aachen.de \\ ${ }^{q}$ Aalborg University, Denmark. E-mail: edc@es.aau.dk
}

\begin{abstract}
As the standardization of full dimension multiple-input multiple-output (MIMO) systems in the $3^{\text {rd }}$ Generation Partnership Project progresses, the research community has started to explore the potential of very large arrays as an enabler technology for meeting the requirements of $5^{\text {th }}$ generation systems. Indeed, in its final deliverable, the European 5G project Mobile and Wireless Communication Enablers for the 2020 Information Society (METIS) identifies massive MIMO as a key 5G enabler and proposes specific technology components that will allow the cost efficient deployment of cellular systems taking advantage of hundreds of antennas at cellular base stations. These technology components include handling the inherent pilot-data resource allocation trade-off in a near optimal fashion, a novel random access scheme supporting a large number of users, coded channel state information for sparse channels in frequency division duplexing systems, managing user grouping and multi-user beamforming, and a decentralized coordinated transceiver design. The aggregate effect of these components enables massive MIMO to contribute to the METIS objectives of delivering very high data rates and managing dense populations.
\end{abstract}

Keywords - multiple antenna systems, channel state information, beamforming, power control, pilot contamination

\section{INTRODUCTION}

Multiple-input multiple-output (MIMO) systems involving a number of antenna elements with an order of magnitude larger than in the early releases of wireless standards is a quickly maturing technology. Indeed, an ongoing work item of the $3^{\text {rd }}$ Generation Partnership Project (3GPP) for Release-13 of the Long Term Evolution (LTE) is identifying the technology enablers and performance benefits of deploying up to 64 antenna ports at wireless access points and base stations (BS) [1]. While this is a significant increase of the number of antenna ports as compared with today's typical deployments, to fully realize the promises of scaling up MIMO to very large (massive) arrays in practice requires further research and system development work [2]. Recent developments by the industrial and academic research communities in related fields such as 3D MIMO, hybrid beamforming (BF) based on combining analog and digital precoding techniques and understanding the asymptotic behavior of random matrices suggest that massive MIMO can bring unprecedented gains in terms of spectral and energy efficiency and robustness to hardware failures and impairments. Also, as it was pointed out in [3], higher frequency bands, like millimeter wave (mmWave), are appealing for large-scale antenna systems, since the physical array size can be greatly reduced due to the decrease in wavelength.

The METIS technology components (TCs) address two essential issues in massive MIMO: channel state information (CSI) acquisition and transceiver structure [6]. CSI acquisition in massive MIMO is challenging because of the many channel links that need to be estimated and the problem of pilot contamination. Likewise, the very large number of channel links represents one major impediment in transceiver design as it sharply increases the computational complexity, calls for robust designs against CSI errors to achieve the desired gains and increases the traffic data transport over the backhaul in coordinated systems.

The massive MIMO TCs in METIS address two major 5G goals defined in the project: a) the ability to deliver very large data rates to each user, b) the ability to deliver a high quality of service to a very dense population of users. Note that goal b) is rarely addressed, while it is becoming more and more relevant in view of the capability of massive MIMO to spatially multiplex a large number of users. Furthermore, the METIS technology components target legacy bands below $6 \mathrm{GHz}$. This focus is justified by the allocation of frequency bands below 6 $\mathrm{GHz}$ by the recent International Telecommunications 
Union World Radio Conference WRC-15, while for backhaul.

higher frequency bands no allocations for $5 \mathrm{G}$ have been made so far.

The next section discusses CSI acquisition techniques. Section III discusses transceiver design aspects, while While time division duplexing (TDD) is the widely Section IV offers concluding remarks and proposes topics preferred solution for massive MIMO systems, as it for future works.

scales with the number of antennas at the BS, frequency division duplexing (FDD) remains an attractive solution for operators. Therefore, METIS developed TCs both for TDD and FDD systems.

In TDD systems, one of the main impediments specific to massive MIMO is pilot contamination [2], [4], [5]. CSI is acquired via uplink training that is used for downlink beamforming relying on channel reciprocity. In multicell multiuser massive MIMO systems, pilot sequences are typically reused in neighbor cells due to the large number of simultaneously served users and the constraints on pilot sequence lengths. The effect of pilot contamination on the performance of massive MIMO systems, particularly on the achievable rates, has been analyzed by a great number of contributions and efficient mitigation schemes have been proposed [4], [5].

In this paper, we discuss a low-rate multi-cell coordination scheme that allows for pilot sequence reuse across neighbor cells and balances the pilot-data power trade-off. Pilot sequence reuse can avoid pilot contamination among neighboring cells and thereby avoid the most severe effects of interference among pilot signals. Pilot-data power balancing, as an additional technology component, can achieve near optimal rate performance in multicell systems [8]. Furthermore, for a scenario with a massive density of devices and intermittent traffic, we argue that a centralized pilot assignment becomes infeasible and propose a decentralized assignment based on coded random access at the devices, along with a data communication protocol. In FDD systems, the main impediment is the scalability of CSI acquisition with the number of antennas. In this paper, we argue that exploiting the sparse properties of the channel in urban environments and using the concept of coded CSI reporting can provide a solution.

For multi-cell multi-user massive MIMO systems, we propose low complexity transceiver designs that well approximate the performance of centrally coordinated schemes. The large density of users is addressed by devising a user clustering and grouping mechanism enabling an independent user selection and precoding per-group. In principle, multi-cell coordinated transceiver design imposes a heavy traffic load on the backhaul due to the large number of channel links. Those constraints are relaxed relying of large scale system analysis which enables a design based on local CSI and the exchange of long term statistics on the

\section{Channel State InFormation ACQUisition}

\section{A. Low Rate Multi-cell coordination for pilot contamination mitigation in TDD systems}

Understanding and managing the inherent trade-offs related to CSI acquisition in massive MIMO systems is fundamental to the design of such systems [4], [5], [7]. As it has been discussed in details in [4], the level of pilot contamination in terms of the number of users using non-orthogonal pilot sequences can be controlled by, among other techniques, setting the pilot sequence reuse factor across the cells of a multi-cell system. Also, the effect of pilot contamination can be mitigated by downlink pilot contamination elimination precoding (PCEP) proposed by [5]. In our approach, the level of pilot contamination is controlled by adaptively setting the pilot reuse factor and balancing the pilot-data power ratio, which is beneficial for both the uplink and downlink performance.

When operating in limited coherence time and frequency channel conditions, the number of symbols that is available within the coherence time of the channel is limited and the inherent trade-offs between allocating resources to pilot and data symbols include the following:

- Allocating more power, time, or frequency resources improves the quality of the channel estimate, but leaves fewer resources for uplink and downlink data transmission.

- Constructing longer pilot sequences helps to avoid tight pilot reuse in multi-cell systems, which in turn helps to reduce or avoid pilot contamination. On the other hand, spending a greater number of symbols on pilots increases the pilot overhead.

- In multiuser MIMO systems, increasing the number of orthogonal pilot sequences may increase the number of spatially multiplexed users, since a larger number of orthogonal pilot sequences enable the system to distinguish a larger number of users in the code domain. However, this comes at the expense of having longer pilot sequences.

The METIS massive MIMO concept combines the coordinated allocation of resources available for pilot (reference) signals across multiple cells and the balancing of the pilot-data resources within each cell [8]. For pilot contamination mitigation, the Operation and Maintenance $(\mathrm{O} \& \mathrm{M})$ System employs low-rate multi- 
cell coordination to set the pilot reuse factor (e.g. pilot contamination that is seen as a collision in a random reuse-1 or pilot reuse-3) depending on the cell load and access protocol and solved accordingly using novel the coherence time budget that can be used for creating techniques from the area of coded random access [9].

pilot sequences.

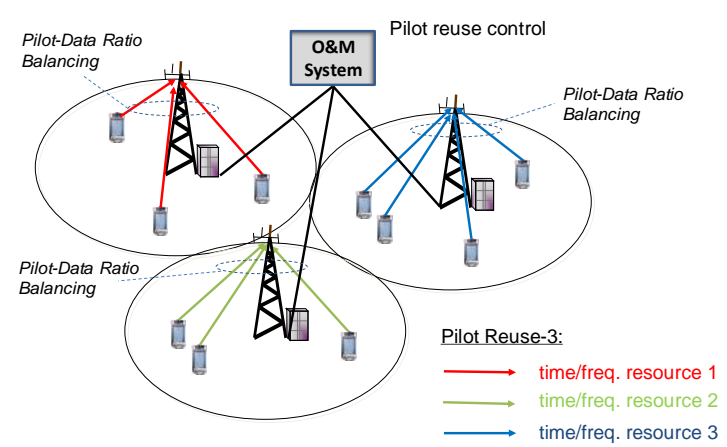

Figure 1: Pilot (reference signal) reuse management by the operation and maintenance $(O \& M)$ system and balancing the pilot-data balance in multi-cell massive MIMO systems

For example, at pedestrian speed of $1.5 \mathrm{~m} / \mathrm{s}$ and outdoor cell radius of around $1000 \mathrm{~m}$ at $2 \mathrm{GHz}$ carrier frequency, the number of symbols within the coherent bandwidth and time are around $\mathrm{B} \mathrm{T}=300 \mathrm{KHz} \times 25$ $\mathrm{ms}=7500$, whereas this coherent budget is only a few hundred symbols at vehicular speeds (with coherence time of $\mathrm{T} \approx 1.25 \mathrm{~ms}$ ).

Notice that even a greater-than-one pilot reuse scheme does not eliminate pilot contamination, since pilot interference can be caused by all surrounding cells. Therefore, within each cell, each mobile station employs pilot-data power ratio balancing at a fine time scale to maximize spectral efficiency [8].

\section{B. Random Pilot Access for Crowd Scenarios in TDD Systems}

One of the main METIS objectives has been to drastically increase the area spectral efficiency that is the bit rate per unit area [6]. Thanks to the very large number of antennas at the BS, a massive MIMO system has the potential to serve a very high density of users and thereby help achieve the area spectral efficiency goal. In such a crowd scenario, the number of orthogonal pilots is much smaller than the number of users. In addition, non-streaming applications are considered that are characterized by intermittent and unpredictable traffic. Those features prohibit a centralized pilot assignment to the users so that random access to the pilot sequences by the users becomes a natural solution.

In the METIS solution, random pilot access is coupled with data transmission in the uplink of a TDD system. Random pilot access brings a new perspective on pilot
We target multi-cell scenarios, machine-type communications and also $5 \mathrm{G}$ scenarios as defined in METIS, where new types of massive array deployment have been determined for hotspots. One example has very large arrays deployed along the infrastructure of a stadium. In such scenarios, pilot contamination becomes an intra-cell interference problem, while in the literature, it has been typically defined as an inter-cell interference problem, which is less easily manageable.

To understand the proposed solution, consider the random access system with a time slotted transmission schedule shown in Table I. In each time slot, each user is active with probability $p_{a}$. An active user chooses a pilot sequence at random from a set of size $\tau$ and transmits it followed by a data transmission. A user may be active in multiple time slots, where the data is retransmitted as a repetition code until the end of the transmission frame.

When two or more users apply the same pilot sequence in the same time slot, the channel estimate will be contaminated. More specifically, an estimate of the sum of the involved channel vectors is achieved. Instead of discarding contaminated channel estimates, they are applied as matched filters on the received data signals.

As a simple example, consider time slot 1 in Table I and noise-free reception. User 1 and User 4 (with channels $h_{i}$ and data $x_{i}(\mathrm{i}=1, \mathrm{i}=4)$ ), collide: the channel estimate corresponding to the transmission of pilot $s_{1}$ is $h_{1}+h_{4}$. Applying this contaminated estimate as a matched filter in the training and data domain, we get two filtered signals: $\left(h_{1}+h_{4}\right)^{H}\left(h_{1} s_{1}+h_{3} s_{2}+h_{4} s_{1}\right)=\left(\left\|h_{1}\right\|^{2}+\left\|h_{4}\right\|^{2}\right) s_{1}$ and $\quad\left(h_{1}+h_{4}\right)^{H}\left(h_{1} x_{1}+h_{3} x_{3}+h_{4} x_{4}\right)=\left\|h_{1}\right\|^{2} x_{1}+$ $\left\|h_{4}\right\|^{2} x_{4}$. The second equation assumes orthogonality between user channels (correlation is considered as interference). Similarly, time slot 3 gives us $\left\|h_{1}\right\|^{2} s_{2}$ and $\left\|h_{1}\right\|^{2} x_{1}$, when applying the matched filter given by the transmission of $s_{2}$. Through simple subtraction, we can now cancel the interference from user 1 in the signals from time slot 1 , in order to recover the message from user 4.

\begin{tabular}{|l|c|c|c|c|c|c|}
\cline { 2 - 7 } \multicolumn{1}{c|}{} & \multicolumn{2}{c|}{ Time slot 1 } & \multicolumn{2}{c|}{ Time slot 2 } & \multicolumn{2}{c|}{ Time slot 3 } \\
\hline User 1 & $s_{1}$ & $x_{1}$ & & & $s_{2}$ & $x_{1}$ \\
\hline User 2 & & & $s_{1}$ & $x_{2}$ & & \\
\hline User 3 & $s_{2}$ & $x_{3}$ & & & $s_{1}$ & $x_{3}$ \\
\hline User 4 & $s_{1}$ & $x_{4}$ & $s_{2}$ & $x_{4}$ & & \\
\hline User 5 & & & $s_{2}$ & $x_{5}$ & $s_{1}$ & $x_{5}$ \\
\hline
\end{tabular}

Table I: Example of uplink transmission during 3 time slots using coded random access with a set of 2 pilot sequences $s_{1}$ and $s_{2}$. 
It has been shown that carefully designed coded full rank sparse matrix for any potential combination of random access systems are asymptotically optimal in the the sparse sub-beams.

sense that they achieve the throughput of fully scheduled and thereby interference free operation. Numerical evaluations show that the proposed solution achieves $45 \%$ of the throughput of scheduled operation with 400 antennas at the base station. For comparison, a scheme reminiscent of the conventional method of slotted ALOHA achieves 33\%. With 1024 antennas at the base station, $61 \%$ is achieved. Therefore, unifying the powers of massive MIMO and coded random access is a promising way to mitigate pilot contamination in scenarios with high user density.

\section{Coded CSI for Sparse Channels in FDD Systems}

In order to harvest the potential gains of massive MIMO technology in frequency domains below $6 \mathrm{GHz}$ (which is considered as the borderline to cmWave systems in the 3GPP), massive MIMO should be facilitated for TDD as well as FDD systems to cover the available paired and unpaired frequency bands. CSI acquisition in FDD systems poses new challenges compared to the typically assumed reciprocity based channel estimation concepts [10]. In METIS, this challenge is addressed by employing coded CSI that takes advantage of the sparsity of the channel as described below.

FDD requires the explicit estimation of downlink (DL) channel components (CC) and the reporting of the estimated CSI using a feedback channel. The wellknown grid of beams (GoB) concept can be advantageously used to subdivide the cells into radial subsectors by a limited set of fixed beams. The key observation is that employing GoB at the BS limits the number of effective wireless channels and thereby the overhead for CSI estimation and reporting. Specifically, the number of $\mathrm{CC}$ that are within a certain power window of, for example, $20 \mathrm{~dB}$ with respect to the strongest $\mathrm{CC}$ are only a small subset of all CCs; typically, 10 to 20 out of overall several hundreds of potential CCs. Thus, the overall channel matrix, containing the CSI between all BS antenna elements and served user equipment (UE) will be sparse.

Our proposed coded CSI concept allows UEs to estimate the UE individual subsets of, for example, the 20 relevant CCs out of 100 or more CCs in total. Each UE estimates and reports the CSI for its sparse set of relevant $\mathrm{CCs}$ to the $\mathrm{BS}$, which combines this information to an overall sparse channel matrix. A Vandermonde like beam specific pilot sequence (= code) is applied to each CC - or, equivalently, Tx-beam. These non-orthogonal sequences provide, for each UE, a

The benefit of using non orthogonal sequences is the high number of supported beams with a limited number of orthogonal pilot resources. Despite an overhead of less than 5 to $10 \%$ an average normalized mean square error below 20dB [6] could be achieved. Together with channel prediction per effective $\mathrm{CC}$ as a further $5 \mathrm{G}$ pillar such low CSI estimation errors will help to approach the performance gains that can be obtained with perfect CSI.

\section{TRANSCEIVER DESIGN}

A. Joint User Clustering, Grouping and MultiUser Beamforming for High User Density

\section{Scenarios}

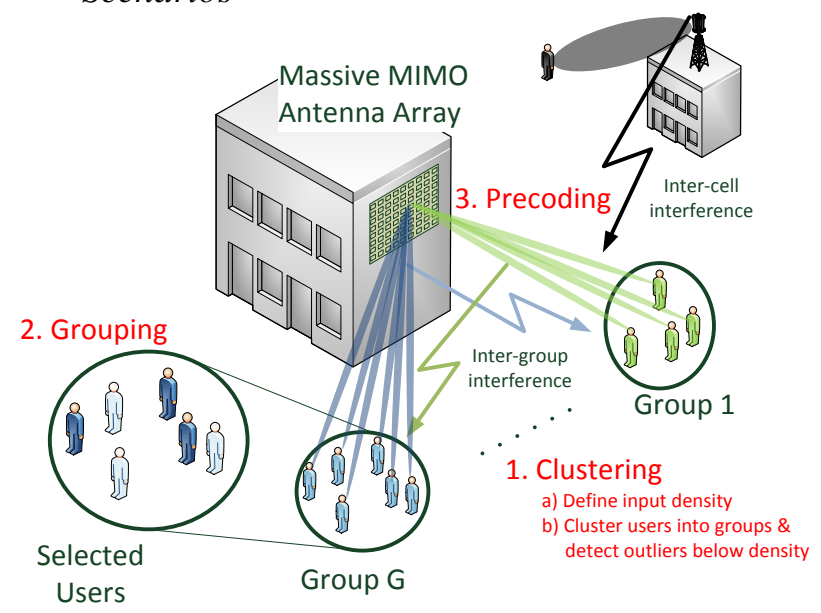

Figure 2: User clustering, grouping and beamforming. A group of served users are similar in terms of second order statistics that can be advantageously used in the precoding step.

Massive MIMO promises spectral efficiency gains by spatial multiplexing of multiple devices [2]. This increased number of devices results in higher complexity and increased signalling or feedback overhead. Thus, new concepts, taking into account user clustering, grouping and beamforming are required. For example, Joint Spatial Division and Multiplexing (JSDM), illustrated in Figure 2, has been proposed to relax feedback and signalling requirements in FDD systems [11].

Therein a two-stage beamforming scheme is used to decouple user-clusters (= groups) from each other such that independent user selection and precoding per group are enabled. The original JSDM scheme proposed in [11] has been designed for an FDD system, and inter-cell interference was not considered by assuming only a single BS. The novelty of the METIS TC, compared to the original JSDM scheme, is the adaptation to TDD mode by including inter-cell interference (ICI) feedback 
from the users, and thereby enabling ICI aware precoding. Accordingly, a two-stage beamforming process embedded in the first and third steps of the METIS concept is proposed:

1. The first step of user clustering divides all users connected to a BS into groups with similar second-order channel statistics. We adapted the density-based spatial clustering of applications with noise (DBSCAN) algorithm to cluster an adaptive number of groups with respect to a certain density (see Reference [EKS+96] in [6]). DBSCAN is a partial clustering mechanism implying that all users without suitable neighbors are discarded into a "noise" group. From this the first-stage (second order statistics) beamformer is derived to spatially separate the user groups and allow independent processing per group, using eigenbeamforing or block diagonalization.

2. In the second step, the user selection is done for each group independently and any multi-user precoding scheme can be applied. Due to the similar channel properties per group (characterized by the same dominant eigenvectors of the channel covariance matrix, as in [11]) an intuitive good solution is semiorthogonal user selection (SUS) as in [12] to find a user subset for spatially multiplexed downlink transmission. In Reference [12], the SUS algorithm is adapted with the maximum sum-rate objective using rate approximation. This ensures that the sum-throughput is increased while the limited transmit power budget is divided among all active spatial data streams.

3. In the third step, the precoding weights for simultaneous multi-user transmission are obtained based on the effective channel (the product of the channel and the first step precoder) Due to ICI from BSs with a lower number of antennas -- as with current LTE base stations or small cells --, a subset of the users is interference limited. Thus, ICI has to be considered in the precoder design to balance signal, inter beam (multi-user) interference and ICI power such that a certain metric is optimized. We use regularized zero-forcing precoding where inter-cell interference is considered in the regularization values. Therefore, we introduced an additional feedback consisting of a scalar (assuming single antenna users) broadband power-value as a coarse quantization of the interferencecovariance matrix (receive covariance matrix subtracted by signal power) measured at the users and feedback to the BS, see [12] for more details.
It was shown that by combining these three steps, large performance gains in sum-throughput in the order of 10 times can be achieved using a 256 antenna array compared to a baseline LTE-A scenario with 8 antennas, see [6] for the detailed scenario assumptions. The gains of user grouping and ICI aware precoding from step 2 and 3 without clustering are given in [12]. It is noteworthy, that the only additional overhead compared to other TDD massive MIMO schemes is the wideband power value proposed in step 3 .

The main finding of this section is that user clustering with traditional algorithms like K-means (see the reference [AV07] in [6]) is not practical since the number of groups is required as an input parameter, which is not known a-priori. An exhaustive search over all possible combinations of user clustering is hardly feasible due to complexity reasons.

Therefore, a more advanced clustering algorithm is required where the clustering of groups depends on the data structure, such as density. We propose the DBSCAN algorithm where a target density is required to form a cluster defined by the minimum number of neighbors within a certain radius and used as input. These parameters can be adapted by the system on the current user distribution.

\section{B. Decentralized Coordinated Transceiver Design}

As a consequence of the potential advantage offered by coordinated multipoint (CoMP) [13], different CoMP variants have been included for the downlink of LTE Advanced systems, such as coordinated beamforming (CB) and joint transmission (JT). With CB, each BS serves the users in its cell by considering the interference generated to the users in other cells. On the other hand, for JT, all users in the cluster are served by all the cooperating BSs, which have available data to the users. Ideally, to enable coordinated beamforming, CSI of all the users in the cluster has to be exchanged between the cooperating BSs. This imposes constraints on the capacity and the delay of the backhaul, which may not be achievable in practice.

Theoretically, in a multi-cell scenario with a very large antenna array at the BS, the inter-cell interference (ICI) can be entirely eliminated by using simple maximum ratio transmission. In practice, however, the maximum number of antennas at each BS is limited. Consequently, interference-aware precoder design with inter-node cooperation should be applied for optimal handling of the remaining ICI [14]. In minimum power beamforming, the optimal decentralization can be realized by exchanging instantaneous locally acquired CSI or terms related to the ICI values via a backhaul link [14]. 
To decrease the load on the backhaul links, the results loss to each user. The local DL channel knowledge is from random matrix theory can be utilized to develop acquired from UL pilots assuming TDD channel approximately optimal beamforming algorithms based reciprocity. The correlation among channel entries $(i, j)$ on uplink-downlink duality, as carried out in [15] for between BS- $b$ and User- $k$ is captured using a simple independent and identically distributed channels. The exponential model $\left[\boldsymbol{\theta}_{b, k}\right]_{i, j}=\rho^{|i-j|}$, where $\boldsymbol{\theta}$ is the algorithm proposed in [6] gives the approximately channel covariance matrix and $\rho$ represents the optimal dual uplink power allocations and the correlation coefficient (assumed to be 0.8 in the numerical corresponding downlink beamformers for the general correlated channel. The method relies only on local CSI knowledge along with channel statistics exchanged among coordinating BSs. Still, the error in approximations causes variations in the resulting SINR values which violate the target SINR feasibility.

Another approach proposed in [6] is to utilize optimization decomposition to decouple the centralized problem to BS-specific subproblems by considering the ICI as the principal coupling parameter among BSs. Furthermore, the ICI terms coupling the coordinating BSs can be approximated via large system analysis such that the ICI terms depend only on the statistical properties of the channel vectors. The large dimension approximation for the ICI terms provides an approximately optimal distributed algorithm that gives the locally feasible beamformers based on the exchanged channel statistics (large-scale fading characteristics and antenna correlation). Only a limited cooperation between nodes is required to share the knowledge about channel statistics among coordinating BSs.

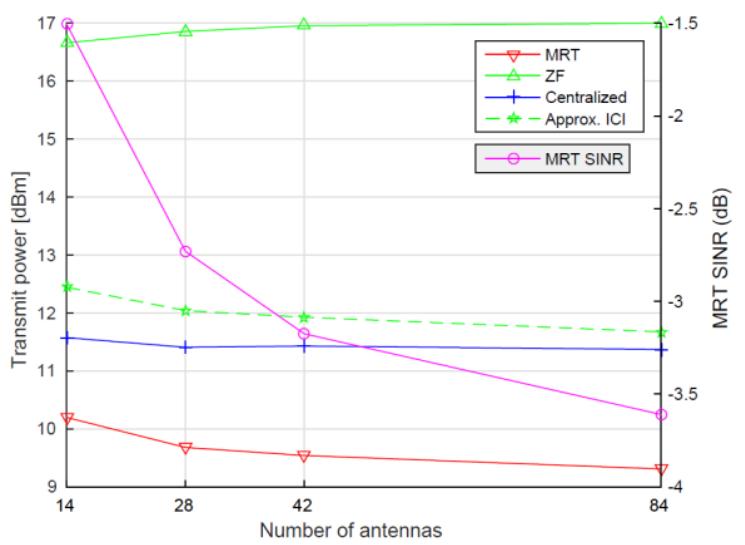

Figure 3: Comparison of the required relative transmit power for $0 \mathrm{~dB}$ SINR target. MRT ignores interference and yields poor SINR (right hand side axes), whereas the other schemes meet the $0 \mathrm{~dB}$ SINR target.

example of Figure 3). More elaborated models have been used in [6] and the references therein.

Figure 3 shows the transmit power versus the number of antennas for $0 \mathrm{~dB}$ SINR target. The algorithm based on ICI approximation is compared to maximum ratio transmission (MRT), zero forcing and optimal centralized beamforming averaged over 1000 random drops. The number of antennas at each BS is varied from $N=14$ to $N=84$ while the total number of users is equal $K=N / 2$. Thus, the spatial loading is fixed as the number of antennas is increased. The results demonstrate that the gap between the proposed approximated ICI algorithm and the optimal case (denoted as centralized) diminishes as the number of antennas and users increase. The relatively small gap even with small dimensions indicates that the approximated algorithm can be applied to the practical scenarios with a limited number of antennas and users.

Figure 3 further demonstrates that both the centralized algorithm and the approximated ICI algorithm greatly outperform the zero forcing beamforming and the performance gap is constant as both $N$ and $K$ are increased. The gap is mainly due to the fact that the zero forcing algorithm wastes degrees of freedom for nulling the interference towards the distant users while both the approximated and the optimal centralized algorithm find the optimal balance between interference suppression and maximizing the desired signal level. MRT obviously requires the least power since the interference is completely ignored at the transmitter. However, as Figure 3 shows, the achievable SINR at the receiver is greatly below the SINR target values due to uncontrolled interuser interference.

\section{IV.CONCLUDING REMARKS}

Scaling up MIMO to hundreds of antenna elements promises unprecedented increase in spectral and energy efficiency. While current standardization efforts target up to 64 antenna ports, the research community, including the METIS project, are developing specific technology components that will allow for several hundreds of antennas at BSs and wireless access points.

A cellular network with 7 cells is considered. In this In this paper we highlighted technology components that example, users are equally distributed between cells and provide solutions to the problems of balancing the an exponential path loss model is used to assign the path inherent trade-off between pilot and data resources in 
multicell systems, acquiring CSI for dense user advantageously combined with the decentralized populations and in FDD systems, scalable beamforming coordinated transceiver design. Determining the exact to a large number of users and multicell operation with combination of the proposed TCs in specific deployment limited backhaul resources. These TCs are solution scenarios is left for future works.

approaches to the problems of CSI acquisition in dense

user populations and scalable transceiver designs for a These ideas and concepts will help massive MIMO to live large number of BS antennas. These TCs can be up to the promises and expectations on the capability of deployed separately or in suitable combinations (Figure delivering very high data rates to each user in high user 4). For example, the joint user clustering and grouping density situations. In fact, the results of METIS suggest scheme facilitating DL beamforming can be combined that massive MIMO is a useful TC not only for creating with the coded random access based uplink scheme to high capacity access links, but also for boosting the support very high density of users. Also, the multicell capacity of backhaul links in dense deployment scenarios. uplink pilot and data power control scheme can be

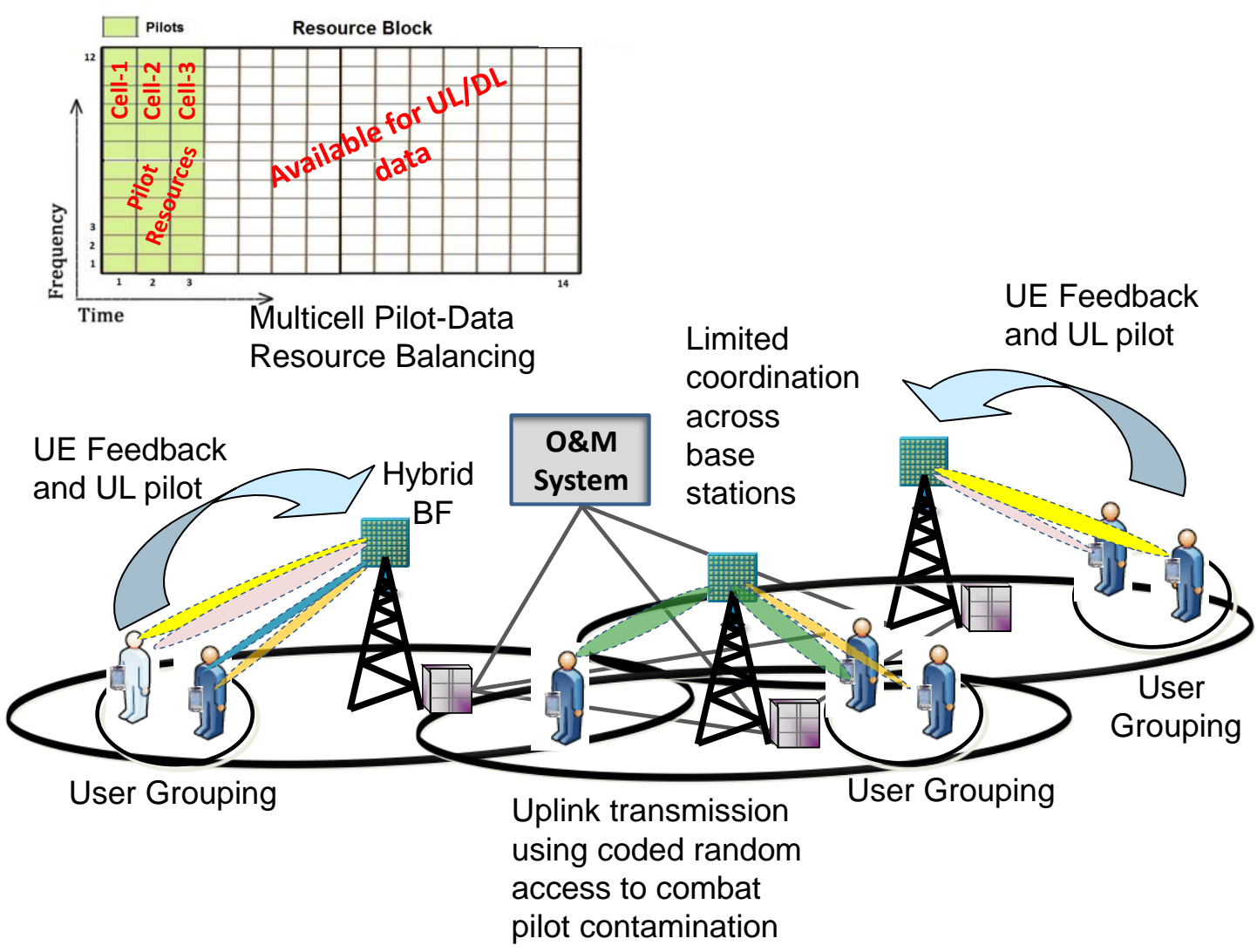

Figure 4 The METIS technology components - CSI based precoding, pilot-data resource balancing, user grouping, limited multicell coordination and uplink transmission using coded random access - can be integrated or used separately in future massive MIMO systems.

\section{REFERENCES}

1. $3 \mathrm{GPP} \quad$ TR $36.897 \quad 3 \mathrm{GPP} \quad$ TR 36.897 V13.0.0, "Study on Elevation Beamforming, Full Dimension (FD) Multiple Input Multiple Output (MIMO) for LTE", Release 13, June 2015.

2. F. Rusek, D. Persson, B. K. Lau, E. G. Larsson, T. L. Marzetta, O. Edfors, and F.
Tufvesson, "Scaling Up MIMO Opportunities and Challenges with Very Large Arrays", IEEE Signal Processing Magazine, Volume 30, No. 1, pp. 40-60, January 2013.

3. Shuangfeng Han, Chih-lin I, Zhikun Xu and Corbett Rowell, "Large-scale Antenna Systems with Hybrid Analog and Digital Beamforming for Millimeter Wave 5G", IEEE Communications Magazine, Vol. 53, 
Issue 1, pp. 186-194, January 2015.

4. Olakunle Elijah, Chee Leow, Tharek Rahman, Solomon Nunoo, Solomon Zakwoi Iliya, "A Comprehensive Survey of Pilot Contamination in Massive MIMO 5G System", IEEE Communications Surveys and Tutorials, Vol.18, Issue 2, pp. 905-923, November 2015.

5. Binyue Liu, Syong Cheng and Xiaojun Yuan "Pilot Contamination Elimination Precoding in Multi-cell Massive MIMO Systems", IEEE Annual International Symposium on Personal, Indoor, and Mobile Radio Communications (PIMRC), pp 320-325, August-September 2015.

6. METIS Deliverable D3.3, "Final Performance Results and Consolidated View on the Most Promising MultiNode/Multi-Antenna Transmission Technologies, February 2015. Available at:http://metis2020.com/documents/deliver ables.

7. N. Jindal and A. Lozano, "A Unified Treatment of Optimum Pilot Overhead in Multipath Fading Channels", IEEE Transactions on Communications, Vol. 58 , No. 10, pp. 2939-2948, October 2010.

8. K. Guo, Y. Guo, G. Fodor, and G. Ascheid, "Uplink Power Control with MMSE Receiver in Multi-cell MU-Massive-MIMO Systems," IEEE International Conference on Communications (ICC), Sydney, Australia, June 2014.

9. J.H. Sørensen, E. de Carvalho, and P. Popovski, "Massive MIMO for Crowd Scenarios: A Solution Based on Random Access", IEEE International Workshop on Massive MIMO: From Theory to Practice, IEEE Global Telecommunications Conference (Globecom), 2014.

10. E. Björnson, E. G. Larsson and T. Marzetta, "Massive MIMO: Ten Myths and One Critical Question", IEEE Communications Magazine, pp. 114-123, February 2016.

11. A. Adhikary, J. Nam, J.-Y. Ahn, and G. Caire, "Joint Spatial Division and Multiplexing - The Large-Scale Array Regime," IEEE Transactions on Information Theory, Vol. 59, No. 10, pp. 6441-6463, Oct 2013.

12. M. Kurras, L. Thiele and T. Haustein, "Interference Aware Massive SDMA with a Large Uniform Rectangular Antenna Array", European Conference on Networks and Communications (EuCNC), pp. 1-5, Bologna, Italy, June. 2014.

13. D. Gesbert, S. Hanly, H. Huang, S. Shamai Shitz, O. Simeone, and W. Yu, "Multi-Cell MIMO Cooperative Networks: A New Look at Interference," IEEE J. Select. Areas Commun., vol. 28, no. 9, pp. 1380 1408, 2010.

14. A. Tölli, H. Pennanen, and P. Komulainen,
"Decentralized Minimum Power Multi-cell Beamforming with Limited Backhaul Signalling", IEEE Trans. on Wireless Comm., Vol. 10, No. 2, pp. 570 - 580, February 2011.

15. S. Lakshminarayana, M. Assaad, M. Debbah, "Coordinated Multicell Beamforming for Massive MIMO: A Random Matrix Approach", IEEE Transactions on Information Theory, Vol. 61, No. 6, pp. 3387-3412, June 2015. 